\title{
Monte Carlo Study of the Interaction Volume Changes by the Beam Skirt in VP-SEM
}

\author{
M. El Azzouzi ${ }^{a, b}$, L. KhouchaF ${ }^{a, *}$ AND A. Achahbar ${ }^{b}$ \\ ${ }^{a}$ Institut MinesTelecom, Lille University, 941 rue Charles Bourseul, CS10838 Douai, France \\ ${ }^{b}$ Condensed Matter Group, Department of Physics, Faculty of Sciences, B.P. 2121, Tetouan, Morocco \\ (Received February 13, 2017; in final form October 4, 2017)
}

In this work we present a new contribution for tracking the behavior of electron beam in gas and then in material placed in the chamber of a variable pressure scanning electron microscope using Monte Carlo simulation. Firstly our results for width and depth of interaction volume in high vacuum mode are compared and are consistent with those obtained by several relationships present in literature. Carbon and aluminum are considered as examples in order to establish the reliability of our approach and experimental data available from the literature. Then, we compared the evolution of width in both high $\left(\operatorname{Re}_{(H V)}\right)$ and low $\left(\operatorname{Re}_{(L V)}\right)$ vacuum modes with enlargement of skirt $\left(R_{s}\right)$. The present work demonstrates that the best resolution conditions for energy, pressure and material, is given by $R_{s}=\operatorname{Re}_{(H V)}$. Finally, the energy that must be used to get the best image resolution for given pressure and material is determined.

DOI: 10.12693/APhysPolA.132.1393

PACS/topics: VP-SEM, skirt, interaction volume, high vacuum, low vacuum, Monte Carlo simulation

\section{Introduction}

Variable pressure scanning electron microscope technology has been largely improved during the last years. In contrast, some fundamental questions are still not well understood concerning the consequences of the interaction between the primary electron beam and the gas. Moreover, there is not yet any analytical method characterizing the electron beam during analysis of nonconductive materials by introducing a quantity of gas in specimen chamber. It is of great importance to know the magnitude and the extent of beam broadening scattering electrons (skirt) and how closely the scattered fraction of electrons can affect the imaging and the spatial resolution of X-ray microanalysis in VP-SEM. Indeed, many works have studied the problems caused by the skirt phenomenon [1-11]. The most of the earlier studies on the topic were dedicated essentially to measure the amplitude of deflected beam [12-14] and to evaluate its impact on the analysis. Recent works propose some experimental equipment and methods to minimize the effect of the gas interaction electron beam and to study the dielectric samples [15]. The interaction was restricted to the primary electron beam with atoms/molecules of gas. Consequently, the interaction volume due to the presence of the skirt, which is the key parameter for spatial resolution, is not considered. Recent studies [16] have compared the skirt radius with X-ray emission volume radius and the generation volume radius of the backscattered electrons in the material calculated from the Monte Carlo computation (CASINO V2.42 software) in high vacuum mode.

\footnotetext{
* corresponding author; e-mail: lahcen.khouchaf@mines-douai.fr
}

In this work, a contribution based on Monte Carlo simulation model to study the effect of skirt on width of interaction volume in gaseous environment (see Fig. 1) by monitoring the behavior of electrons in gas and in material at the same time is developed. Our results have been compared with theoretical values in literature for high vacuum mode that give depth and width of electrons penetration in solid [17-21].

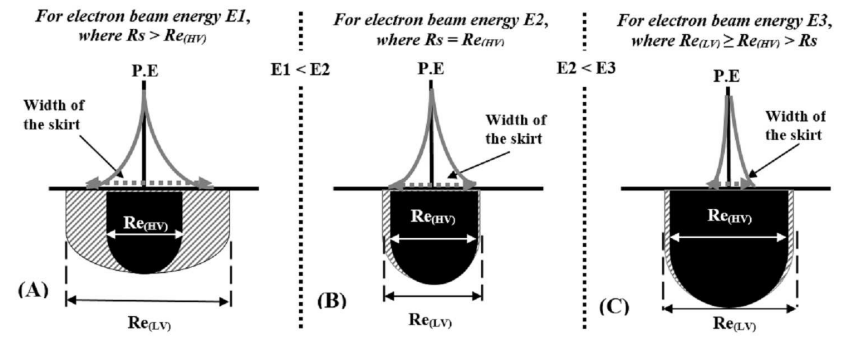

Fig. 1. Schemas showing the relationship between the interaction volume under high vacuum mode $\left(\operatorname{Re}_{(H V)}\right)$ and in low vacuum mode $\left(\operatorname{Re}_{(L V)}\right)$ versus the energy and the skirt $\left(R_{s}\right)$.

Figure 1 shows three cases corresponding to three values of the energy of the electron beam E1, E2, and E3 $(\mathrm{E} 1<\mathrm{E} 2<\mathrm{E} 3)$. In fact, at high vacuum mode, when the incident beam energy increases, the width of interaction volume $\operatorname{Re}_{(H V)}$ increases too but at low vacuum mode, the width of interaction volume has no trivial behavior versus the skirt. It depends on energy and pressure values as we will present in Sect. 3. In regime (A), for electron beam energy $\mathrm{E} 1$, where $R_{s}>\operatorname{Re}_{(H V)}, \operatorname{Re}_{(L V)}$ is determined by scattered electrons beam, because the number of the scattered electrons is much more important than the number of unscattered ones. The size of the skirt and the width $\operatorname{Re}_{(H V)}$ are smaller than the width $\operatorname{Re}_{(L V)}$ 
$\left(\operatorname{Re}_{(L V)}>R_{s}>\operatorname{Re}_{(H V)}\right)$. In regime (B), for electron beam energy E2, where $R_{s}=\operatorname{Re}_{(H V)}$, unscattered and scattered electrons fractions have almost the same effect on the interaction volume. The increase of the number of unscattered electrons decreases both $\operatorname{Re}_{(L V)}$ and $R_{s}$ $\left(\operatorname{Re}_{(L V)} \geq R_{s}=\operatorname{Re}_{(H V)}\right)$. In regime $(\mathrm{C})$, for electron beam energy E3, where $\operatorname{Re}_{(L V)} \geq \operatorname{Re}_{(H V)}>R_{s}$, interaction volume $\operatorname{Re}_{(L V)}$ is governed by unscattered electron beam since the number of these electrons is more important than the scattered ones.

\section{Monte Carlo approach}

Monte Carlo simulations are well suitable for modeling diffusion of highly random particles trajectories. Here, we use Monte Carlo method to study the behavior of incident electrons in gas following each collision until electrons leave the gas and reach the material surface.

\subsection{Theoretical backgrounds}

\subsubsection{Electrons-gas interaction}

This section gives a brief overview of physical processes that occur when an electron beam interacts with gas, and how Monte Carlo method can be used to simulate these processes.

As an electron travels through the gas, considered as a dilute solid, its energy decreases. Since scattering event is energy dependent, the instantaneous energy at any time needs to be calculated. The energy loss is given by [22]:

$$
\frac{\mathrm{d} E}{\mathrm{~d} s}=-78500\left(\frac{\rho Z}{A E}\right) \log \left(\frac{1.166 E}{J}+1\right)\left[\frac{\mathrm{keV}}{\mathrm{cm}}\right]
$$

where $\rho, A$, and $Z$ are respectively, the density, atomic weight, and atomic number of gas. $E$ is the energy of the electron beam in $\mathrm{keV}$ and $J$ is the mean ionization potential in $\mathrm{keV}$, and it can be deduced from the expression [22]:

$$
\left\{\begin{array}{l}
J=11.5 Z[\mathrm{eV}] \text { for } Z \leq 12 \\
J=9.76+58.5 Z^{-0.19}[\mathrm{eV}] \text { for } Z \geq 13
\end{array}\right.
$$

The mean free path of electron $\lambda$ which is the average distance between successive collisions, is given by [23]:

$$
\lambda=\frac{1.38 \times 10^{-23} T}{\sigma P}[\mathrm{~m}] .
$$

$T$ is the absolute temperature of gas in $\mathrm{K}, P$ is the gas pressure $[\mathrm{Pa}], \sigma$ is the total scattering cross-section $\left[\mathrm{m}^{2}\right]$ and it varies with the electron energy. Values of total scattering cross-section for various types of gas are derived from the work of Danilatos [24]. Knowing the elastic mean free path between the collisions, the distance between them $\mathrm{Sn}$, can be computed using the relation [25]:

$$
S_{n}=-\lambda \ln (R N D) \text {. }
$$

$R N D$ is pseudo random number in $[0,1]$

The new direction of the electron after the $n$-th collision is defined by two angles $(\theta, \Psi)$. Calculation of these two angles gives us the new direction of the electron. Angular elastic deflection $\theta$, which is the angle between the direction of incident electron and the direction after its collision, is given by the screened elastic Rutherford scattering [26]:

$$
\cos \theta=1-\frac{2 \alpha R N D}{1+\alpha-R N D}
$$

$\alpha$ is the screening factor computed from [27]:

$$
\alpha=0.00343 \frac{Z^{0.67}}{E} \text {. }
$$

Azimuthal scattering angle [25] determined in perpendicular plane to the electron direction is obtained by simple selection of a random number RND.

$$
\Psi=2 \pi R N D \text {. }
$$

Energy loss $\Delta E$ along the step is calculated using Eq. (1) and Eq. (4) as

$$
\Delta E=S_{n}(\mathrm{~d} E / \mathrm{d} S) .
$$

Initial coordinates of electron at the entrance are $x_{0}=0$, $y_{0}=0, z_{0}=0$. Once electron interacts with target at point $A_{1}$, it changes direction and goes into the volume of gas while losing part of its energy. If the electron undergoes an interaction at point $A_{2}$ after travelling the path $S_{n}$, coordinates of point $A_{2}$ are calculated in relation to those of $A_{1}$ and are given by [26]:

$$
\left\{\begin{array}{l}
x_{n}=x_{n-1}+S_{n} C A, \\
y_{n}=y_{n-1}+S_{n} C B, \\
z_{n}=z_{n-1}+S_{n} C C .
\end{array}\right.
$$

$C A, C B$ and $C C$ are direction cosines

$$
\left\{\begin{array}{l}
C A=C_{x} \cos \theta+V_{1} V_{3}+C_{y} V_{2} V_{4} \\
C B=C_{y} \cos \theta+V_{4}\left(C_{z} V_{1}-C_{x} V_{2}\right) \\
C C=C_{z} \cos \theta+V_{2} V_{3}-C_{y} V_{1} V_{4}
\end{array}\right.
$$

$C_{x}, C_{y}$, and $C_{z}$ take respectively values 0 , and 1 before the first collision

$$
\begin{aligned}
& V_{1}=A N \sin \theta ; V_{2}=A N A M \sin \theta ; V_{3}=\cos \psi ; \\
& V_{4}=\sin \psi ; A N=-\left(\frac{C_{x}}{C_{z}}\right) ; A M=\frac{1}{\sqrt{1+A N^{2}}} .
\end{aligned}
$$

$A N$ and $A M$ are parameters introduced in order to simplify the writing of formulas of $V_{1}$ and $V_{2}$ when changing variables.

\subsubsection{Electrons-matter interaction}

In matter, this work is based on plural scattering model introduced by [25-28], which was specifically designed to produce good data in short computing times on small computers. We consider that every electron travels exactly the same total path length in specimen before it stops [25]. This distance is found by numerically evaluating the integral

$$
R_{B}=\int_{0}^{E}\left(\frac{-1}{\mathrm{~d} E / \mathrm{d} S}\right) \mathrm{d} E .
$$

The total distance $R_{B}$ is often called the Bethe range. In our simulations, it was divided into 50 steps of equal length [25].

At the beginning of each step $i, E_{i}$ is computed from $E_{i-1}$ as

$$
E_{i}=E_{i-1}-\int\left(\frac{\mathrm{d} E}{\mathrm{~d} S}\right) \mathrm{d} S,
$$

where $E_{1}$ is set equal to incident beam energy $E_{0}$ and $E_{51}$ is set equal to zero. 
Randomized Rutherford type scattering formula is used to determine scattering angle [29]:

$$
\tan \left(\frac{\theta}{2}\right)=\tan \left(\frac{\theta_{0}}{2}\right)\left(\frac{E_{0}}{E}\right)\left(\frac{1}{\sqrt{R N D}}-1\right),
$$

where

$$
\tan \left(\frac{\theta_{0}}{2}\right)=\frac{0.0144 Z}{2 p_{0} E_{0}}
$$

and

$$
p_{0}=0.394 * Z^{0.4} / E \text {. }
$$

$\theta$ represents scattering angle, $\theta_{0}$ - the minimum scattering angle, $E$ is the instantaneous energy of electron (in $\mathrm{keV}$ ) and $E_{0}$ is the incident beam energy and $p_{0}$ is the maximum impact parameter. The relation between $\tan \left(\frac{\theta_{0}}{2}\right)$ and backscattering coefficient $\eta$ can be written as a polynomial

$$
\begin{aligned}
& \tan \left(\frac{\theta_{0}}{2}\right)=0.016697+055108 \eta \\
& -0.96777 \eta^{2}+1.8846 \eta^{3} .
\end{aligned}
$$

Backscattering coefficient can be computed with the formula [30]:

$$
\eta(Z, E)=E^{m(z)} C(z),
$$

where

$$
\begin{aligned}
& m(z)=0.1382-\frac{0.9211}{\sqrt{Z}}, \\
& C=0.1904-0.2235(\ln Z)+0.1292(\ln Z)^{2} \\
& \quad-0.01491(\ln Z)^{3} .
\end{aligned}
$$

The procedure is to find a value for $\eta_{\text {mix }}$ (for a mixture of elements) using the relation

$$
\eta_{\text {mix }}=\sum_{i=1}^{i=n} C_{i} \eta_{i},
$$

where $C_{i}$ are the concentrations of elements and $\eta_{i}$ are found from Eq. (17). Similarly, azimuthal angle is given by the same expression as previously used (Eq. (7)).

The ultimate stage of our simulations is the deduction of maximum width and maximum depth as illustrated in Fig. 2.
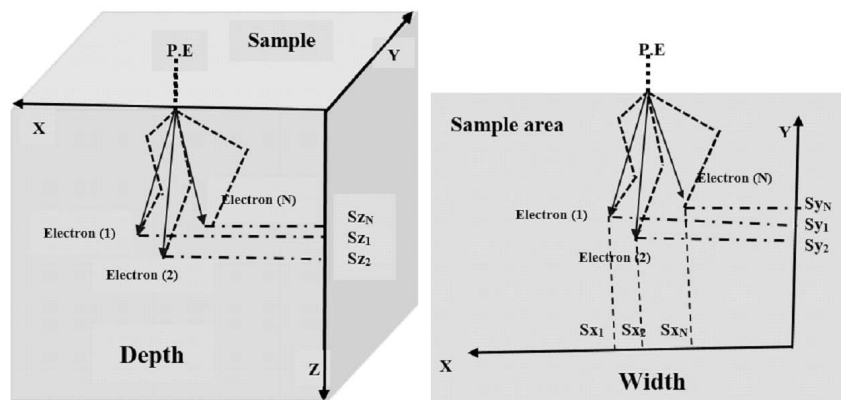

Fig. 2. Schematic representation of the width and the depth calculation method.
The average maximum depth is

$$
R_{p}=\frac{\sum_{i=1}^{N} S z_{i}}{N} .
$$

The average maximum width is

$$
R_{e}=\frac{\sum_{i=1}^{N} S_{i}}{N},
$$

where $S_{i}=\sqrt{S x_{i}^{2}+S y_{i}^{2}}$.

\subsection{Analytical expressions in literature}

Our Monte Carlo results have been compared with several semi-empirical expressions in literature for both maximum depth $\left(R_{p}\right)$ and maximum width $(\mathrm{Re})$. For $R_{p}$, we use the expressions proposed by

- Wittry and Kyser [21]:

$$
R_{p}[\mu \mathrm{m}]=\left(\frac{25.6}{\rho}\right)\left(\frac{E_{0}}{30}\right)^{1.68} ;
$$

- Everhart and Holf [17]:

$$
R_{p}[\mu \mathrm{m}]=\left(\frac{0.0398}{\rho}\right) E_{0}^{1.75} \text {. }
$$

When $5 \mathrm{keV} \leq E \leq 25 \mathrm{keV}$ and $10<Z<15$,

- Kanaya-Okayama [19]:

$R_{p}[\mu \mathrm{m}]=\left(\frac{0.0276 A}{\rho Z^{0.889}}\right) E_{0}^{1.67}$,

- Potts [20]:

$R_{p}[\mu \mathrm{m}]=\frac{0.1}{\rho E_{0}^{1.5}}$.

$A$ is the atomic weight $[\mathrm{g} / \mathrm{mol}], E$ is the energy of the incident beam $[\mathrm{keV}], \rho$ is the density of material $\left[\mathrm{g} / \mathrm{cm}^{3}\right]$ and $Z$ is its atomic number.

For $R_{e}$, we use for comparison, the expressions proposed by

- Goldstein [18]:

$R_{e}[\mu \mathrm{m}]=\frac{0.625 Z}{E_{0}}\left(\frac{\rho}{A}\right)^{0.5} x_{0}^{1.5}$,

- Potts [20]:

$R_{e}[\mu \mathrm{m}]=\frac{0.77}{\rho} E_{0}^{1.5}$.

$x_{0}$ is in $\mu \mathrm{m}, E_{0}$ is in $\mathrm{keV}$ and $\rho$ is in $\mathrm{g} / \mathrm{cm}^{3}$.

Physical properties of materials used in simulations are shown in Table I.

TABLE I

Physical properties of materials used.

\begin{tabular}{c|c|c|c|c|c|c|c}
\hline \hline & \multicolumn{3}{|c|}{ PMMA } & \multicolumn{2}{c|}{ Silica } & $\mathrm{Al}$ & $\mathrm{C}$ \\
\cline { 2 - 6 } & $\mathrm{C}$ & $\mathrm{O}$ & $\mathrm{H}$ & $\mathrm{Si}$ & $\mathrm{O}$ & & \\
\hline atomic number & 6 & 8 & 1 & 14 & 8 & 13 & 6 \\
\cline { 2 - 5 } density $\left[\mathrm{g} / \mathrm{cm}^{3}\right]$ & \multicolumn{3}{|c|}{1.188} & 2.648 & 2.7 & 2.26
\end{tabular}




\section{Results and discussion}

For each material, Monte Carlo simulations is carried out to calculate the depth and the width of the interaction volume, in the energy ranging from 5 to $30 \mathrm{keV}$. The number of simulated electrons is 10000 and the simulated electron beam is impinging at normal incidence. For each phase, twenty simulations is carried out to calculate average of maximum width and maximum depth of interaction volume. $90 \%$ of the scattered electrons (low vacuum mode) are considered to calculate the skirt radius and the width of interaction volume [31].

\subsection{High vacuum mode}

Our results are compared with Eqs. (23)-(28) as shown in Fig. 3 and Fig. 4 and are consistent with theoretical predictions [17-21]. Concretely, in the case of maximum depth (Fig. 3), our findings are very close to those given by Kanaya-Okayama [19], which are the most widely used in literature. In the case of maximum width (Fig. 4), our results are located between the other curves but qualitatively more consistent with the Potts equation [20].
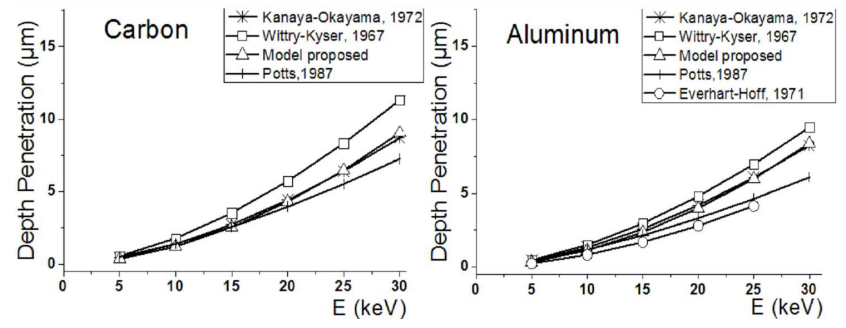

Fig. 3. Comparison of average of maximum penetration depth obtained by Monte Carlo simulation results and formulae (23)-(26) as function of energy for aluminum and carbon.
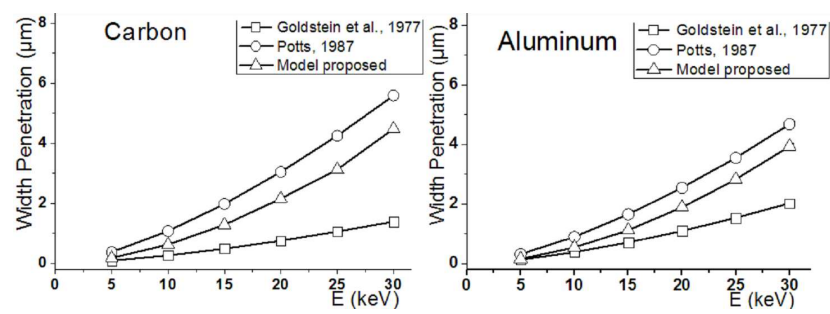

Fig. 4. Comparison of average of maximum penetration width obtained by Monte Carlo simulation results and formulae (27)-(28) as function of energy for aluminum and carbon.

\subsection{Low vacuum mode: effect of the skirt on the interaction volume}

One advantage of using VP-SEM in low vacuum mode is to observe materials that are not stable under high vacuum mode conditions. In counterpart, it presents a serious disadvantage regarding the resolution in $\mathrm{X}$-ray microanalysis and the image quality. The goal of this work is then to search the experimental conditions that give the best possible resolution.

Lateral resolution in high vacuum mode in CSEM is essentially limited by the size of interaction volume inside the material. Under low vacuum mode, spatial distribution of incident electron beam (skirt), due to the presence of the gas, can affect badly lateral resolution in X-ray microanalysis. Searching for optimal parameters to limit degradation of the resolution, we have performed a comparative analysis between lateral electron distributions obtained at high and low vacuum modes as function of the skirt radius, by choosing water vapor $(Z=7.42)$ as standard gas in the specimen chamber with pressure value 0.1 and 0.5 Torr. The working distance is taken equal to $2 \mathrm{~mm}$ for calculating $R_{s}$ and $\operatorname{Re}_{(L V)}$.

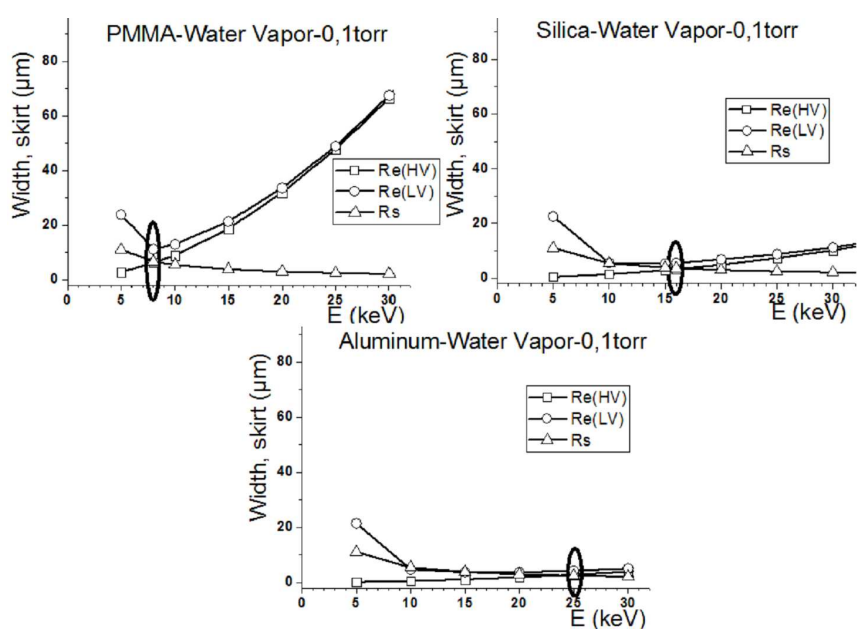

Fig. 5. Evolution of the skirt $\left(R_{s}\right)$ and the width of interaction volume in high vacuum mode $\left(\operatorname{Re}_{(H V)}\right)$ and low vacuum mode $\left(\operatorname{Re}_{(L V)}\right)$ for PMMA, silica and aluminum as function of energy.

Figure 5 shows the three situations schematized in Fig. 1. In the case of PMMA material, the first regime is when $E<8 \mathrm{keV}$ and the width of interaction volume in high vacuum mode $\operatorname{Re}_{(H V)}$ is less than $R_{s}$ and $\operatorname{Re}_{(L V)}$ and consequently $\operatorname{Re}_{(L V)}>R_{s}>\operatorname{Re}_{(H V)}$. In fact, the interaction volume under low vacuum mode is more important than the corresponding one under high vacuum mode which is only created by primary electron beam. In the second regime, $E>8 \mathrm{keV}, \operatorname{Re}_{(H V)}$ and $\operatorname{Re}_{(L V)}$ are greater than $R_{s}\left(\operatorname{Re}_{(L V)} \geq \operatorname{Re}_{(H V)}\right)$. In this case, the skirt radius does not affect interaction volume $\operatorname{Re}_{(L V)}$ since the number of scattered electrons due to the collisions with molecules of gas are weakest compared to unscattered ones. The third regime is the intersection point (bold circle in Fig. 5) between the two tendencies cited above, i.e., when $E=8 \mathrm{keV}$. Here, $R_{s}=\operatorname{Re}_{(H V)}$, and interaction volume is affected by both scattered and unscattered electrons beams because their effects are similar $\left(\operatorname{Re}_{(L V)} \geq \operatorname{Re}_{(H V)}\right)$. Thus, for PMMA at 0.1 Torr, the skirt affects the width $\operatorname{Re}_{(L V)}$ for energies less than $8 \mathrm{keV}$. For the other materials, we observe similar be- 
havior. For silica, a dense material with higher atomic number than PMMA, the intersection point is shifted to $E=16 \mathrm{keV}$. For aluminum, denser material compared to PMMA and silica, the point of intersection is about $25 \mathrm{keV}$.

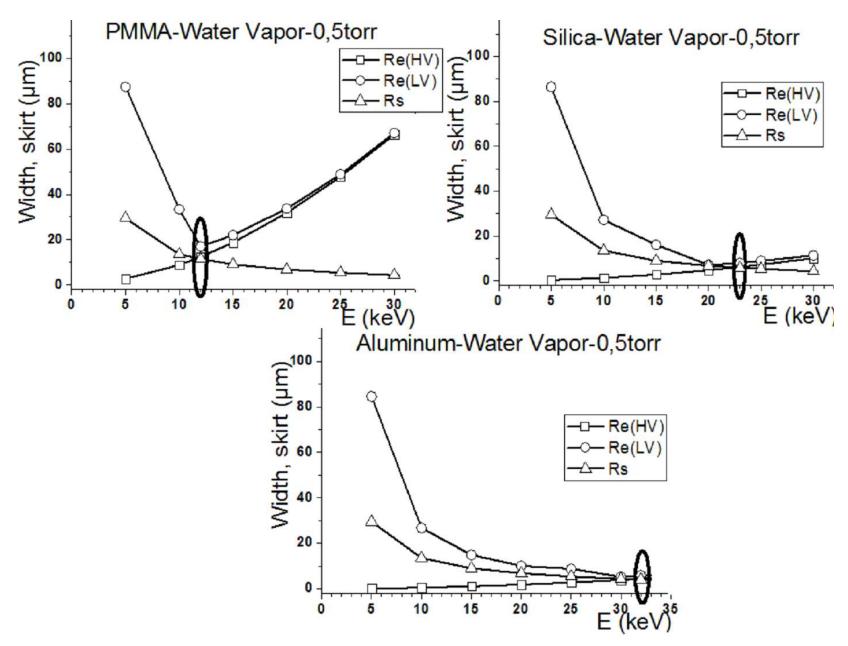

Fig. 6. Evolution of skirt $\left(R_{s}\right)$ and width of interaction volume in high vacuum $\left(\operatorname{Re}_{(H V)}\right)$ mode and low vacuum mode $\left(\operatorname{Re}_{(L V)}\right)$ for PMMA, silica, and aluminum as a function of energy.

In Fig. 6 there are shown the width of interaction volume under high and low vacuum modes and the skirt radius for 0.5 Torr gas pressure. Indeed, in agreement with previous study (Khouhaf et al. 2004), the increase in gas pressure increases considerably the number of scattered electrons in the specimen chamber, which makes the first regime $\left(\operatorname{Re}_{(L V)}>R_{s}>\operatorname{Re}_{(H V)}\right)$ larger and shifts the intersection point mentioned above at higher values. As consequence, the new intersection points are $12 \mathrm{keV}$ for PMMA, $23 \mathrm{keV}$ for silica and $32 \mathrm{keV}$ for aluminum. Then, the extent of each regime depends on atomic number, density of material, and gas pressure. By comparing results in Fig. 5 and Fig. 6, we can affirm that the condition $R_{s}=\operatorname{Re}_{(H V)}$ is the best choice for an optimum resolution in X-ray microanalysis, since it represents the equilibrium between degradation of the image due to the charge accumulation effect when $\operatorname{Re}_{(L V)} \geq \operatorname{Re}_{(H V)}>R_{s}$, and bad image resolution when $\operatorname{Re}_{(L V)}>R_{s}>\operatorname{Re}_{(H V)}$.

Using this criteria, the optimum energy for best resolution as function of pressure for the three different materials is calculated (Fig. 7).

In fact, we can see that the intersection point is shifted with increasing atomic number $Z$ of the specimen and with increasing pressure $P$ of the gas. The results in Fig. 7 give us the way the intersection point changes with the atomic number of material as a function of pressure. The energy of the primary beam that allows the best resolution increases proportionally with pressure. Above approximately 1 Torr, we observe that the intersection point remains almost constant with pressure especially for PMMA.

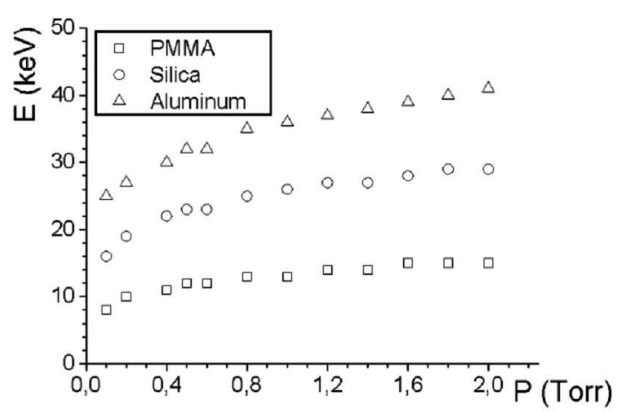

Fig. 7. The optimum energy for the best imaging resolution as function of the pressure for PMMA, silica, and aluminum for water vapor. To add values of 0.1 and 0.5 Torr.

\section{Conclusion}

A Monte Carlo approach to quantify the effect of electron scattering by gas in an VP-SEM is proposed in order to find the best condition for an optimum resolution in X-ray microanalysis. Simultaneously electron-gas and electron-material interactions using different materials and water vapor as gas are considered. In high vacuum mode, our results are very similar to the known theoretical predictions, especially to the Kanaya-Okayama equation in the case of depth of interaction volume. Under low vacuum conditions, we find that the best results are obtained by choosing the energy of electron beam that corresponds to the condition $\operatorname{Re}_{(H V)}$. In fact, it is shown that this energy increases with atomic number of material and with pressure of gas.

\section{References}

[1] A.N. Farley, J.S. Shah, J. Microsc. 158, 379 (1990).

[2] D.C. Siegee, C. Gilpin, Scan. Microsc. Suppl. 8, 219 (1994).

[3] G.D. Danilatos, Microchim. Acta 114, 143 (1994).

[4] C. Gilpin, D.C. Siegee, J. Microsc. 179, 28 (1995).

[5] R. Gauvain, Scanning 21, 388 (1999).

[6] C. Mathieu, Scan. Microsc. 13, 23 (1999).

[7] J.F. Mansfield, Mikrochim. Acta 132, 137 (2000).

[8] L. Khouchaf, J. Verstraete, J. Phys. IV 12, 341 (2002).

[9] L. Khouchaf, J. Verstraete, J. Phys. IV 118, 237 (2004).

[10] L. Khouchaf, F. Boinski, Vacuum 81, 599 (2007).

[11] L. Khouchaf, C. Mathieu, A. Kadoun, Vacuum 86, 62 (2011).

[12] E. Dohene, Scanning 19, 75(1997).

[13] S.A. Wight, Scanning 23, 320 (2001).

[14] C. Arnoult, J. Di Martino, L. Khouchaf, V. Toniazzo, D. Ruch, Micron 42, 877 (2011).

[15] W. Słówko, M. Krysztof, Acta Phys. Pol. A 120, 23 (2011).

[16] A. Zoukel, L. Khouchaf, C. Arnoult, J.Di. Martino, D. Ruch, Micron 46, 12 (2013). 
[17] T. Everhart, P. Hoff, J. Appl. Phys. 42, 5837 (1971).

[18] J.I. Goldstein, J.L. Costley, G.W. Lorimer, S.J.B. Reed, Scan. Electr. Microsc. 1, 315 (1977).

[19] K. Kanaya, S. Okayam, J. Phys. D Appl. Phys. 5, 43 (1972)

[20] P.J. Potts, A Handbook of Silicate Rock Analysis, Springer, Dordrecht 1987.

[21] D.B. Wittry, D.F. Kyser, J. Appl. Phys. 38, 375 (1967).

[22] D.C. Joy, S. Luo, Scanning 11, 176 (1989).

[23] G.D. Danilatos, Foundations of Environmental Scanning Electron Microscopy. Advances in Electronics and Electron Physics, Academic Press, Boston 1988.

[24] G.D. Danilatos, Micron 45, 1 (2013).
[25] D.C. Joy, Scan. Microsc. 5, 329 (1991).

[26] D.E. Newbury, H. Yakowitz, in: Use of Monte Carlo Calculations in Electron Probe Microanalysis and Scaning Electron Microscopy, Eds. K.F.J. Heinrich, D.E. Newbury, H. Yakowitz, NBS Special Publication No. 460, 1976.

[27] H.E. Bishop, in Ref. [26], p. 5.

[28] L. Curgenven, P. Duncumb, Tube Investments Research Labs Report, 303 (1971).

[29] G. Love, M.G.C. Cox, V.D. Scott, J. Phys. D Appl. Phys. 10, 7 (1977).

[30] H. Hunger, L. Küchler, Physica Status Solidi A 56, K45 (1979).

[31] O. Mansour, K. Aidaoui, A. Kadoun, L. Khouchaf, C. Mathieu, Vacuum 84, 458 (2010). 